\title{
La grande misère des humbles gens dans La Grande Maison de Mohamed Dib \\ Par
} Amin Salah El-Din Amin Mohamed*

\section{Résumé :}

dr_amin56@yahoo.com

La littérature algérienne d'expression française d'avant l'indépendance se développe dans un contexte particulier marqué par le colonialisme. Elle dénonce le système impérialiste non seulement français mais occidental dans son ensemble. Elle exprime des thèmes spécifiquement algériens : l'identité nationale algérienne par la description d'une réalité socioculturelle qui allait à l'encontre des clichés habituels de l'exotisme, l'exil physique et linguistique, la réhabilitation de l'homme algérien, la situation coloniale. Elle dévoile l'exploitation de l'indigène par le colon, sa destitution par la force des armes de son statut d'être humain, pour se voir ravalé à un simple outil économique au profit de son exploiteur. Mon étude est basée sur l'étude romanesque dans la littérature algérienne d'expression française dans le but de naviguer profondément dans la société algérienne durant la période coloniale par les idées nouvelles de l'écrivain.

\section{Résumé de l'œuvre de Mohamed Dib}

Une maison énorme et grouillante comme il s'en trouve tant dans les villes algériennes. La faim animale, la panique, la générosité, la gentillesse, le bonheur d'un cadeau reçu (surtout si c'est quelque chose à manger) tissent le drame quotidien de ces existences qui, dans ce cadre sordide et tumultueux, demeurent insaisissables aux étrangers. Omar, le petit héros de ce roman, n'est pas un observateur impersonnel et froid - on s'en doute. Dans la souffrance, la violence - et, par làdessous, l'amour - il se hausse à la compréhension de l'événement et de la condition des siens. Nous sommes en 1939, année décisive pour le monde moderne.

* Maître de conférences - Faculté Al-Alsun, Université d'Assouan.

(La grande misère des humbles gens ...)Dr. Amin Salah El-Din 


\section{INTRODUCTION}

S'il y a eu période d'avant littérature africaine avec des auteurs spécialement colons qui travaillaient pour dévaloriser la race noire et l'Afrique, la littérature Africaine d'avant les grandes indépendances [1957-1962] a été à priori celle de la lutte des races d'Afrique pour un affranchissement du pesant joug colonial. Et par moments, il y a eu une classification de cette littérature part zones spatiales ou territoriales, oubliant même que toutes ces littératures, même si elles n'étaient pas reconnues du colon, s'inscrivaient dans un seul but et un même moule : le moule de la reconnaissance de l'Afrique et le désir à l'indépendance du continent asservi depuis le deuxième moitié XIXème siècle. C'est ainsi qu'on entendra parler de littératures négro-africaine, maghrébine, littérature Africaine Anglaise, hispanique ou lusophone et autres. Et l'auteur de notre article est Mohamed Dib dont nous aurons à travailler sue l'œuvre sur le thème est « La grande misère des humbles gens dans La grande Maison».

Cette œuvre romanesque est une présentation réaliste de la société algérienne de la période d'écriture qui expose les souffrances et les misères du petit peuple de cette Algérie en buttes aux problèmes sociaux, politiques et culturels dans un Maghreb en quête de liberté et de mieux être. Mais la situation n'est pas aussi simple que cela devrait l'être à cause du colonialisme et de la situation même de ce vaste pays du désert du Sahara alors département de la France métropolitaine qui impose sa théorie du Maître et de l'Esclave à toutes ses colonies et l'Algérie département français n'y échappe pas. 
Alors, dans une sorte de révolte, et s'érigeant en défenseur de la vérité de tous ces peuples nord-africains, Mohamed Dib écrira cette œuvre sociocritique. Mais parler de la littérature algérienne, c'est considérer le contexte général de l'histoire du Maghreb où beaucoup d'États ont acquis leur indépendance.

Dans cet article portant sur le thème « La grande misère des gens humbles dans La Grande Maison» de Mohamed Dib, nous présenterons d'abord l'auteur, son œuvre, et l'espace et le temps. Ensuite, nous examinerons la condition humaine telle que décrite dans l'œuvre, pour finir par l'analyse de l'expression de la grande misère des humbles gens dans cette œuvre d'exposition d'une Algérie engagée sous la colonisation française.

\section{I- L'AUTEUR, L'GUVRE ET L'ESPACE-TEMPS CHEZ MOHAMED DIB.}

Il sera question ici de la présentation de l'auteur choisi. Aussi, présenterons-nous l'œuvre support de cet article pour finir par l'espace et le temps utilisés par

\section{I-1- MOHAMED DIB, AUTEUR DE LA GRANDE MAISON.}

\section{I-1-1- BIOGRAPHIE MOHAMED DIB.}

De nationalité algérienne, Mohammed Dib est l'auteur du roman La Grande Maison. Né le 21 juillet 1920 dans une famille de la petite bourgeoisie tlmécénienne Mohammed Dib a exercé plusieurs métiers dont le commerce, la menuiserie-ébéniste, courtier en immobilier. Il fait ses études primaires et secondaires en français exclusivement, sans fréquenter l'école coranique comme l'usage le voulait. En 1931, son père décède alors que ses affaires ont périclité, laissant sa famille dans une situation précaire. Sans être 
autobiographiques, ses premiers romans portent l'empreinte de cette période difficile.

En 1940, il est requis civil au Génie militaire à Tlemcen puis, en 1942, interprète-rédacteur français-anglais au Service Prêt-Bail à Alger.

Dès 1948 aux Rencontres de Sidi Madani, organisées près de Blida par les Mouvements de Jeunesse et d'Éducation populaire. Il rencontre Albert Camus, Louis Guilloux, Brice Parain, Jean Cayrol (qui publiera ses premières œuvres au Seuil), du poète Jean Sénac avec lesquels il se liera d'amitié. Ses textes écrits en nouvelles serviront à élaborer ses premiers romans. Mohammed Dib travaille comme journaliste à Alger républicain, journal proche du Parti communiste algérien. Il fait des reportages sur les mouvements sociaux en Algérie, écrit des articles et chroniques culturelles engagés sur les conditions de vie des Algériens sous domination coloniale.

En 1951, il épouse à Alger Colette Bellissant, la fille de son ami Roger Bellissant, un instituteur progressiste de Tlemcen. Il meurt le 2 mai 2003 à l'âge de 82 ans, à La Celle SaintCloud, où il est enterré.

\section{I-1-2- BIBLIOGRAPHIE ET PRIX LITTÉRAIRES DE L'AUTEUR.}

Comme écrivain, Mohammed Dib a reçu de nombreuses récompenses pour son œuvre, notamment le prix Fénéon en 1953 pour son premier roman La Grande Maison, le prix René Laporte en 1962 pour le recueil de poésie Ombre gardienne, le prix De l'Association des Écrivains de Langue Française en 1977 pour le roman Habel, et plusieurs prix de l'Académie française pour la poésie ou les romans. En 1994, 
il reçoit le Grand Prix de la Francophonie décerné par l'Académie française, attribué pour la première fois à un écrivain maghrébin ; en 1998, le prix Mallarmé est attribué à son recueil de poésie L'Enfant jazz et le Grand Prix du Roman de la Ville de Paris à l'ensemble de son œuvre romanesque ; en 2001, le Prix des Découvreurs de la Ville de Boulogne/Mer récompense l'ensemble de son œuvre poétique. Qu'en est-il de l'œuvre en elle-même ?

\section{I-2- DE L'GUVRE LA GRANDE MAISON.}

Paru en première édition 1952 et en seconde édition en 1996 aux Éditions du Seuil à Paris, La Grande Maison est un roman de 192 pages selon l'édition. Il est le premier volet de la trilogie de l'auteur sur l'Algérie. L'écrivain y critique le système colonial français qui réduit les indigènes à une misère sans nom, à une misère déshumanisante (Arnauld, 1986, p. 51). À cette époque, la presse coloniale n'accueille pas favorablement ce premier roman soutenu par la presse libre française. D'ailleurs des auteurs émérites le soutiendront: Louis Aragon et André Malraux. Les deux autres volets, L'Incendie et Le Métier à tisser, paraissent en 1954, année du déclenchement de la guerre d'Algérie, et en 1957. Les trois romans sont le témoignage de la grande misère des villes et des campagnes sous la colonisation française, des grèves des ouvriers agricoles, des revendications nationalistes naissantes. Il publie en 1956 son premier recueil de nouvelles, $A u$ Café, chez Gallimard, puis en 1958 aux éditions La Farandole, Baba Fekrane, un album de contes pour enfants. Il a aussi des recueils de poèmes. 


\section{I-3- L'ESPACE ET LE TEMPS DANS L'GUVRE DE MOHAMED DIB.}

La littérature maghrébine a toujours eu pour but d'être le reflet réel de la société, mais aussi, un moyen pour faire connaître et raconter le malheur du peuple maghrébin. C'est donc une littérature d'éveil des consciences par son engagement politique, social, culturel. C'est dans ce cadre général que s'inscrit depuis les années cinquante, la littérature algérienne d'expression française; celle de dénoncer les fléaux du colonialisme. L'espace, c'est l'Algérie miniaturisée par «Dar-Sbitar» (Dib, 1952-1966, p. 6) qui semble symbolique de ce vaste pays. Il y a aussi la cour de l'école et les rues de la ville de Tlemcen où se trouve « DarSbitar »

D'ailleurs; la pédagogue Djoher Amhisis-Oukel, en parle. Pour elle l'espace «Dar-Sbitar» est l'Algérie réelle de la période coloniale comme décrite par l'auteur de La Grande Maison. (Amhisis-Oukel, 2012, p. 43) L'espace de l'ouvrage, c'est «Dar-Sbitar »; une grande maison avec des habitants pauvres et indigents. Le temps se confond à la période coloniale telle que vue par Mohamed Dib. Quant aux personnages actifs, ils sont de diverses variances sociales, mais dans une misère différente : «Omar, Rachid Berri, Halim », «Yamina la bienfaitrice», «Si Salah, le propriétaire », Veste-de-kaki ». (Dib, 1952-1966, pp. 5-8)

C'est pour la raison du choix de «La Grande Maison » qui représente une image fantastique qui décrit une réalité concrète. Cette œuvre, premier roman de l'écrivain algérien le plus connu situe avec précision dans l'espace et le temps, l'action se passe à la veille de la Seconde Guerre mondiale 
dans la ville de Tlemcen où il y a beaucoup d'enfants qui mendient. Il s'agit ici d'un milieu urbain et d'une famille où la survie est particulièrement difficile lorsque la mère, Aïni, se retrouve seule avec ses trois enfants à nourrir après la mort de son mari. Elle travaille tout le temps et avoir essayé plusieurs métiers : "Ä̈ni avait changé plusieurs fois de travail... Elle avait eu, indéniablement beaucoup de métiers.» (Dib, 1952-1966, p. 131) Dans de telles conditions quiconque ne travaille pas est senti par les autres comme une bouche inutile et aucun sentiment de tendresse ou d'amour ne peut survivra à cela.

L'auteur, son œuvre, espace et temps présentés, nous abordons à présent la condition humaine dans l'autre.

\section{II- LA CONDITION HUMAINE DANS LA GRANDE} MAISON.

\section{II-1- BASE ET CONTEXTE DE LA DÉPLORABLE} CONDITION HUMAINE DANS L'CEUVRE ?

La base de cette œuvre, c'est l'exposition et la critique du colonialisme dans une Algérie à travers la littérature d'expression française d'avant l'indépendance qui s'est développée dans un contexte particulier marqué par le colonialisme, la volonté algérienne de liberté. Elle dénonce le système impérialiste franco-occidental général. Elle développe des thèmes spécifiquement algériens liés à l'identité nationale algérienne par la description d'une réalité socioculturelle faite de misère des gens qui ne cherchent que la paix. Aussi, les clichés d'exotisme, l'exil, la réhabilitation de l'homme algérien, mais surtout la situation coloniale, dévoile l'exploitation de l'indigène par le colon. C'est cette 
réalité poignante que l'œuvre critique et met à nu sous la lumière des vérités de l'époque.

Les Algériens sont destitués par la force des armes et ramener à un simple outil économique est bien montré par Mohamed Dib tout au long de cette œuvre. Il faut donc agir comme le stipule la conception sartrienne de la littérature engagée :

«La parole est un certain moment particulier de l'action et ne se comprend pas en dehors d'elle, or, Parler c'est agir. Toute chose qu'on nomme n'est déjà plus la même. Elle a perdu son innocence ». (Sartre, 1948, pp. 71-72)

Il est important de savoir que la notion même de littérature algérienne n'a de sens qu'à partir du moment où on est sorti d'une simple imitation de la littérature française des années 30, pour assister : À la naissance d'une parole «algérienne », voire "maghrébine », au cœur d'une langue que l'Histoire qui leur est imposée. Une langue que ces auteurs vont malaxer, travailler pour l'adapter à leur besoin de dire.

En effet, cette forme de littérature tire son idéologie du passé, de la période coloniale en restant ancrée dans la réalité que les nations maghrébines ont vécu durant cette période.

M. Lacheraf en dit d'ailleurs que :

"Cette littérature, bien qu'imparfaitement, va refléter, pour la première fois dans les lettres françaises une réalité qu'aucun écrivain, que même Camus n'avait eu le courage de traduire». . . (Lacheraf, 1988, p. 119) 
C'est une littérature qui pousse les écrivains à s'extérioriser à travers la langue française, la langue de l'ennemi, c'est en interaction avec leur langue maternelle, la langue arabe. Elle est devenue un témoin fidèle qui reflète la réalité de la société algérienne avant et après l'indépendance. Et Mohamed Dib y puise de la puissance pour exposer la souffrance des habitants de «Dar-Sbitar»; c'est pour cela Gustave Lanson avait raison lorsqu'il dit : «La littérature, est l'expression directe de sa société » (Guatarni, 2010)

De plus, son engagement l'auteur de La Grande Maison fonde ses textes sur la nécessité avec beaucoup de fermeté par sa volonté de "fondre sa voix dans la voix collective » (Dib, Le 4 juin 1964.) et de faire de son œuvre anticoloniale « autant d'armes de combat » (Belhadj-Kacem, 1983, p. 7) pour une littérature nationale.

\section{II-2- CRITIQUE DE LA CONDITION HUMAINE DANS L'CEUVRE?}

Les romans Le fils du pauvre de Mouloud Feraoun (1950), La colline oubliée de Mouloud Mammeri (1952), et La Grande Maison de Mohammed Dib (1952) sont avant tout, considérés une série de reportages peignent avec réalisme les couches sociales musulmanes de l'époque; une forme indirecte de crier à l'injustice, de décrire les conditions catastrophiques que vit la population sous l'étau de la colonisation (Remache, 2018, p. 67). On ne peut pas ignorer les terribles moments de l'histoire algérienne notamment ceux de la période d'occupation française. Plus de cent trente ans que le peuple algérien ploie sous le poids de l'occupation ; ce sont des années de souffrance et misère, de torture d'une 
nation complétement déchirée par de longues années de résignation.

Par son réalisme et son engagement, Mohammed Dib nous a faits connaître l'Algérie originelle dans le monde littéraire durant la colonisation française. Nous avons vu naître une nation dans ses écrits. Dans sa trilogie intitulée Algérie, La Grande Maison ne peut en être détachée, il fait appel à une révolution, qui pousse Jean Déjeux à écrire dans son livre que : "La littérature Algérienne contemporaine de Mohammed Dib a voulu d'abord être témoin de sa société et de son temps ». (Déjeux, 1979, p. 98)

Un grand nombre des écrivains algériens ont parlé de la Révolution algérienne anticolonialiste français. D'ailleurs, peu ont essayé de transmettre l'amertume réelle des familles algériennes durant cette période et la description minutieuse de la misère à la recherche de l'issue, parmi ces auteurs, nous avons Mohamed Dib et son roman intitulé La Grande Maison.

Cet extrait est déjà une critique de cette condition humaine :

Sa mère pensait à Si Salah, le propriétaire, qui avait horreur des enfants de ses locataires. Il leur interdisait de s'amuser dans la cour ; s'il les y surprenait, il les bousculait et houspillait leurs parents. Ceux-ci n'avaient jamais le courage de lui répondre; quand ils le voyaient, ils se figeaient dans une attitude humiliée ou se réfugiaient dans leurs chambres. En face du propriétaire ils se sentaient envahis par le respect où les jetait une crainte sans bornes. En l'absence de Si Salah, sa femme, vieille à figure chafouine, les assaillait de ses cris d'orfraie. 
L'image donnée dans l'œuvre par le romancier à travers l'indigence des enfants montre, du peuple algérien qu'il n'est pas une masse statique mais une dynamique nation en gestation :

Il est incontestable, déclarait Dib en 1958, que je traite du peuple algérien. De son réveil jusqu'à maintenant, l'Algérie n'était pas nommée en littérature. Dépeindre un paysage, ceux qui l'habitent, les faire parler comme ils parlent, c'est leur donner une existence qui ne pourra plus être contestée. On pose le problème en posant l'homme [...]. Je vis avec mon peuple. J'ignore tout du monde bourgeois. (Dib, Témoignage Chrétien, 1985)

\section{Il faut à présent aborder un autre aspect de la condition} de vie : la condition des personnages de l'œuvre de Mohamed Dib.

\section{II-3- LES CONDITIONS DE VIE DES PERSONNAGES DANS L'EUVRE.}

Les habitants de La Grande Maison appartiennent tous à la classe des indigènes, mais pire, à la classe indigente des pauvres et vivent dans cette conviction que rien ne peut changer. Mais Omar, tout jeune qu'il est, refuse de se résigner et il est révolté aussi par la résignation des autres autour de lui. Il n'arrive à comprendre ni la situation ni le fait que les victimes acceptent leur sort (Brahimi, 2001, p. 76) comme le pense une auteure qui a lu l'œuvre. Aussi, la réflexion du petit est déjà une réponse cachée du peuple Algérien dont la condition est exposée ici : 
"Mais pourquoi sommes-nous pauvres? Jamais sa mère, ni les autres, ne donnent de réponse. Pourtant, c'est ce qu'il fallait savoir. Parfois les uns et les autres décidaient : C'est notre destin. Ou bien : Dieu sait. Mais est-ce une explication, cela? Omar ne comprenait pas qu'on s'en tint de telles raisons. Non, une explication comme cellelà n'éclairait rien. »

(Dib, La Grande Maison, 1952-1966, p. 117)

La Grande Maison a un nom : «Dar-Sbitar », c'est un gros bâtiment misérable barré d'une grande porte monumentale mais branlante, et entourant une cour où le puits est «bien trop près des toilettes » (Dib, La Grande Maison, 1952-1966, p. 7). Le bâtiment abrite des familles misérables luttant toutes de leur mieux mais sans jamais vaincre, contre la misère et la famine. Dans ce foyer, Dar Sbitar, qui se situe dans un quartier très ancien de la ville de Tlemcen, c'est celui où habite la famille d'une veuve, "Aïni et ses enfants », Omar et ses deux sœurs, et une grand-mère grabataire, «comme tout le monde ici, les uns sur les autres. Dar-Sbitar était pleine comme une ruche » (Dib, La Grande Maison, 1952-1966, pp. 6768). C'est à travers cette famille que l'auteur nous dévoile l'extrême misère de cette société algérienne durant cette période.

Le roman est écrit de point de vue de l'aîné, Omar, partagé à l'égard de sa mère (comme elle l'est elle-même envers lui) entre demande d'amour, détresse et agressivité. On assiste à leur lutte quotidienne pour ne pas mourir de faim et pour ne pas non plus perdre leur dignité. Dib a pour objectif de persuader le lecteur français ou étranger sur la réalité 
coloniale amère. C'est pour cela Jean Sénac avait raison quand il dit: «Memmi, Dib, Mammeri nous émouvaient par une sorte d'autopsie minutieuse, de recensement discret de l'injustice. » (Déjeux, Mohamed Dib, écrivain algérien, 1977, p. 84)

Faisant face aux appels quotidiens de ses enfants, la mère est désemparée. Elle maudit son défunt mari qui est parti se reposer en la laissant dans la grande misère. À tous ces désastres de cette famille, s'ajoute la grand-mère paralytique délaissée par ses enfants, elle est aussi chez sa fille Aïni. C'est un autre cas qui a besoin de pitié :

Quant à Grand-mère, ses trois mois chez Aïni étaient passés depuis longtemps déjà. Grand-mère, à partir de ce moment fut abandonnée à Ä̈ni pour de bon. Ses filles et son fils avaient refusé de la reprendre.

(Dib, La Grande Maison, 1952-1966, p. 68)

Alors à partir de la condition humaine exposée dans cette œuvre de la période coloniale, comment s'exprime la misère des humbles gens?

\section{III- L'EXPRESSION DE LA GRANDE MISÈRE DES HUMBLES GENS.}

\section{III- 1- DESCRIPTION DE LA MISÈRE, DURE ET CRUE.}

Mohamed Dib, en esthète au sein du livre, le fait par l'apparition d'un espoir encore vague et très menacé. L'auteur fait apparaître dans son roman ce qui est aujourd'hui reconnu comme un fait historique, c'est-à-dire la naissance à cette époque du nationalisme algérien, ici représenté par le personnage du militant appelé Hamid Saraj « homme instruit, militant de nationalisme qui lutte à côté des ouvriers et des fellahs pour une amélioration de leurs conditions de vie ». Nourreddine Belhadj-Kacem reprend l'idée de misère de

(La grande misère des humbles gens ...)Dr. Amin Salah El-Din 
l'auteur. L'un des chapitres du roman donne en substance le discours que celui-ci prononce devant une salle pleine de gens, des travailleurs qui vibrent à ses paroles:

«L'homme parle dans le fond de la salle. [...] on entend fort bien les paroles ».

"Les travailleurs de la terre ne peuvent plus vivre avec les salaires qu'ils touchent. Ils manifesteront avec force. L'orateur cite en exemple des domaines que connaissent les fellahs ».

"Il faut en finir, avec cette misère ».

"Les ouvriers agricoles sont les premières victimes visées par l'exploitation qui sévit notre pays. "

(Dib, La Grande Maison, 1952-1966, pp. 115-116)

Dans une interview donnée en 1954, il dit : Faire le tableau d'une misère est une besogne absurde si elle ne permet pas d'esquisser déjà la conscience qui fera craquer cette misère [...]. Nous lui (l'auteur) savons gré d'avoir arraché vigoureusement le voile dont on s'obstine à masquer la détresse de notre pays. (Guatarni, 2010)

Dans la même veine, Albert Memmi a indiqué dans une lettre publiée dans le Monde: Les écrivains maghrébins ont l'expression des communautés qui ont vécu la situation coloniale en colonisés; ils ont annoncé la genèse et la constitution de ces communautés en nations. ( Le Monde, le 6 avril 1965)

Il y a pourtant dans ce livre un grand souffle épique et la volonté d'affirmer que ces gens-là, un jour, auront droit au bonheur et à la dignité. ... reconnu comme un fait historique, c'est-à-dire la naissance à cette époque du nationalisme algérien, ici représenté par un personnage de militant appelé Hamid Saraj. Que ce soit dans La Grande Maison ou

(La grande misère des humbles gens ...)Dr. Amin Salah El-Din 
Dar-Sbitar, dit Mohammed Dib, c'est l'esquisse même de notre Algérie musulmane dans ce qu'elle a d'endémique : la misère et l'ignorance. Le régime colonial français est à la base de toutes les calamités surtout sociales vécues par les Algériens. La colonisation française est donc la cause essentielle de toutes les misères dont souffre le peuple algérien incarné dans La Grande Maison de Dib. Omar, le protagoniste du roman, refuse de se plier à ce système colonial opprimant. Les problèmes dont il souffre ne sont pas isolables de ceux de son peuple.

Dans ce récit, Omar représente donc la «conscience collective ». Pour définir la conscience collective :

Il s'agit en premier lieu de valeurs et de normes qui sont acceptées comme un ensemble par un groupe social et qui déterminent la conscience de chacun des membres du groupe.

(Zima, 1985, p. 25)

La situation coloniale est aussi claire dès le premier chapitre quand s'ouvre la parenthèse en arabe sur la leçon de morale du maître, M. Hassan, sur la patrie. C'est aussi l'arrestation d'Hamid qui tente d'organiser les ouvriers agricoles. L'origine espagnole d'une partie des colons, tel Gonzales le petit patron qui emploie Aïni à coudre des empeignes d'espadrilles. (Dib, 1952-1966, p. 93)

Il est incontestable, déclarait l'auteur en 1958, que je traite du peuple. De son réveil jusqu'à maintenant, l'Algérie n'était pas nommée en littérature. Dépeindre un paysage, ceux qui l'habitent, les faire parler comme ils parlent, c'est leur donner une existence qui ne pourra plus être contesté. On 
pose le problème en posant le problème de l'homme [...] Je vis avec mon peuple. J'ignore tout du monde bourgeois.

En lisant ce roman, nous touchons d'une manière sensible le réalisme que l'écrivain éprouve dans les pages du roman, l'écrivain écrit lorsqu'il se sent pris par le besoin d's'exprimer autrement dit, lorsqu'il a une idée à communiquer aux opinions nationale et internationale. Il écrit pour aborder une question sociale et pour remettre en cause le cours de l'histoire qui défavorise son peuple et tenter, à son niveau, de reconstruire une Algérie fraternelle, viable et vivable, construite par tous et pour tous, pour les Européens comme pour les autochtones.

\section{III- 2- LES DIFFÉRENTS ASPECTS DE LA MISÈRE. III- 2-1- LA MISÈRE DE L'ENFANCE}

C'est quoi la misère ? Du latin miseria, la misère est une condition pénible de nature physique, matérielle ou morale, susceptible d'inspirer la pitié ; un état d'extrême pauvreté, de privation des ressources nécessaires à la vie. La misère est constituée par les réactions engendrées par la douleur et elle est le résultat de phénomènes physiques et psychologiques dans lesquels l'angoisse et les expériences douloureuses vécues tiennent une place considérable.

La misère! Elle est partout. Elle est autour de nous, elle est en nous. Que l'on vienne de n'importe quel milieu de la société, la misère fait partie de l'homme. Elle est capable de prendre toutes les formes imaginables: maladie, torture physique, deuil, famine, souffrance morale, chagrin, occupation...

(Dib, 1952-1966, p. 93) 
Constantin François Volney l'exprime en 1791 à la page 43 d'une de ses œuvres :

«Il [l'homme] apprit à distinguer les plantes utiles des nuisibles, à combattre les éléments, à saisir une proie, à défendre sa vie; et il allégea sa misère » (Volney, 1791, p. 43)

En effet, la misère est un sujet très vaste qui peut être abordé sous différentes approches: des points de vue: social, psychologique, philosophique. Mohammed Dib le démystifie dans La Grande Maison par le mensonge de la France-mèrepatrie des Algériens... La femme-mère abandonnée, affamée, épuisée par le travail et la misère personnifie l'Algérie à la veille de la guerre d'indépendance dans un bulletin. Dans le roman de Mohamed Dib, la misère a pris plusieurs aspects ; la misère de la mère, celle de son fils, celle de sa maman, pire la misère de la patrie.

La misère ici n'est pas une formule ni un thème de méditation. Elle est. Elle crie et elle désespère. Encore une fois qu'avons-nous fait pour elle et avons-nous le droit de nous détourner d'elle? (Dib, 1952-1966, p. 93)

La misère extrême se manifeste par l'omniprésence de la faim qui impose son despotisme sur leur vie quotidienne. Attyka chante aussi «Donnez-moi de l'eau fraîche / Du miel et du pain d'orge » (Dib, La Grande Maison, 1952-1966, p. 52) et plus loin Aouïcha et Mériem les deux sœurs d'Omar songeront à un plat royal: le couscous. Toujours, ils cherchent le pain. Parfois ils ont «recours à l'eau pour se remplir l'estomac» vide. Et quand ce n'est pas la faim, c'est la chaleur estivale qui, jour et nuit, pèse sur ce petit monde, en plus de l'exploitation coloniale. Dans son roman, M. Dib 
nous décrit clairement les situations désespérées de cette communauté qui souffre, et son intention, et sa cible était de persuader le lecteur étranger, français, de l'informer sur une vérité durable, une réalité coloniale.

Le roman s'ouvre sur une scène sensible où les enfants sont réunis. Ce sont «les gosses»; pauvres et misérables de la ville de Tlemcen qui sont «morts de faim». Ils veulent présenter Omar comme chef ; un symbole pour eux. En effet, Omar incarne tous les enfants de l'Algérie ; on ne le trouve que parmi ses pareils. Ces enfants sont présentés comme une synecdoque : les voix et les mains :

"Omar se planta devant Rachid Berri. Il n'était pas le seul, un faisceau de mains tendues s'était formé et chacune quémandait sa part et les voix s'élevèrent en une prière.»

(Dib, 1952-1966, p. 7)

Dans La Grande Maison, se dévoile l'image d'une enfance malheureuse. Tous les enfants souffrent de la même manière et ont un but existentiel: la recherche continuelle d'un morceau de pain. Cela nous amène à dire qu'il s'agit ici d'un récit collectif d'enfance dont Omar est la figure de proue de la société enfantine et sa recherche permanente au pain : « $i l$ exigeait d'eux sa part ». Ce qui par ailleurs attire l'attention, est que l'écrivain parle des enfants des couches sociales différentes à travers une comparaison entre ces enfants qui appartiennent à des environnements différents. On a les écoliers, les copains d'Omar qui sont aussi pauvres et misérables que lui ; il y a un point commun qui les unit, c'est comment trouver de quoi à manger. Aussi, de l'autre côté, il y a un autre milieu d'enfants : ceux qui sont si riches, qui ne 
connaissent pas la faim et la misère ; c'est tout un paradoxe dans un même environnement : l'école... !

On n'avait jamais vu Driss faire le geste de donner: Omar ne comprenait pas pourquoi tous l'entouraient ainsi. Était-ce l'obscur que leur inspirait un être qui mangeait chaque jour à faim? Étaient-ils fascinés par la puissance sacrée, incarnée en cet enfant mou et sot?

(Dib, 1952-1966, p. 13)

Il est clair que l'écrivain veut attirer l'attention sur les contradictions de cette société par ce rapport incongru entre les fils des riches et ceux des miséreux qui cohabitent. Les clivages sont si grands comme le dit Dib qu' " on risquait beaucoup à les attaquer : ceux-là avaient leurs courtisans parmi les élèves et les instituteurs »(Dib, La Grande Maison, 1952-1966, p. 13). Cela signifie que les fils des riches sont entourés d'une protection; c'est celle de la richesse.

Le garçon [Omar] extorque les morceaux de pain à ses camardes, il est aussi capable de le gagner en rendant services à Yamina qui le traite avec tendresse et avec générosité :

Yamina le récompensait à son retour en lui donnant une tranche de pain avec un fruit ou un piment grillé - de temps en temps, un morceau de viande ou une sardine frite. Quelquefois, après déjeuner ou dîner, elle l'appelait... La veuve ne le traitait pas comme un chien; et cela lui plaisait. Ne pas être humilié.

(Dib, 1952-1966, p. 9) 
On retrouve dans chaque page du roman, le héros Omar quémandant le pain. Ainsi telle une malédiction du personnage, le pain devient le seul objectif de la vie, non seulement pour lui mais aussi pour ses compagnons de misère. Les préoccupations des «humbles gens » ne se résumaient alors qu'à avoir la pitance pour se remplir les ventres vides. La faim et le pain sont deux faces de la même médaille : nul, dans cette œuvre sur la condition humaine, n'échappe à ce bréviaire de l'indigence, à cette logique dichotomique voulue par Dib :

«Il avait terriblement faim, toujours, et il n'avait presque jamais rien à manger à la maison; il avait faim au point que certaines fois l'écume de sa salive se durcissait dans sa bouche. Subsister, par conséquent, était pour lui l'unique préoccupation. » (Dib, 1952-1966, p.105)

\section{III-2-2- LA MISÈRE, UNE FORCE EXISTENTIELLE AU FEMININ.}

L'amélioration la réalité quotidienne, dans sa famille, à DarSbitar, dans la rue, est son but initial et final. Omar s'interroge sur le comportement des adultes, sur la présence de la colonie européenne, sur la misère des uns et l'injustice sociale des autres: "Pourquoi sommes-nous pauvres? Jamais sa mère ni les autres, ne donnaient de réponse ». L'écrivain nous incite à contempler l'état lamentable des habitants de Dar-Sbitar dans toute leur humanité : leurs besoins, leurs sentiments et leurs sensations. C'est d'une misère totale qui rend accable cette société à s'exprimée vue la question de la faim et la difficulté à obtenir un morceau de pain : 
«La faim, de plus en plus lancinante, faisant gargouiller les intestins des petits. Timidement d'abord ils demandèrent à manger. Ä̈ni paraissait écrasée. Tous ensemble alors ils implorèrent. »(Dib, 1952-1966, pp.107-108)

La faim représente un axe essentiel à travers les représentations psychologiques que l'écrivain a pu exposer avec soin ; la faim obsède complétement les personnages et les rend violents voire cruels envers soi-même, envers les autres et malpolis à l'égard des adultes :

- Hé, Mama! tonitruait Aïni dans son oreille en poussant vers elle l'écuelle. Tu ne vois pas que je t'apporte à manger? On bien ce que j'apporte te plaît?

La vieille femme ne remuait pas. Ä̈ni se saisissait de l'ustensile puis empoignait la tête de grandmère et lui fourrait l'écuelle sous le nez?

- Oui, ma fille, j'ai vu. Pourquoi me traites-tu comme ça?

- Tiens, mange! lui disait Ä̈ni en la secouant sans ménagement.

- Elle bredouillait quelques mots entre ses dents :

«puisses-tu manger du poison.

(Dib, 1952-1966, pp.125-136)

Aussi, dans l'œuvre, Aïni incarne la femme algérienne, sa situation et son rôle efficace dans la société algérienne, durant la colonisation française en l'Algérie. Elle est une pauvre veuve d'une trentaine d'année, qui occupe une chambre avec sa famille à Dar-Sbitar. Elle est cheftaine de 
famille, celle qui travaille et gagne l'argent pour faire survivre sa famille « Aouicha, Meriem, Omar et la Grandmère Mama ». Elle a deux responsabilités : celle de mère devant ses enfants et au même temps fille responsable de sa mère handicapée. Aïni est le symbole de la femme algérienne pleine de volonté aux émotions à fleur de peau, dont le temps est destiné à assurer l'existence des siens. Elle ne laisse pas une place aux sentiments affectueux, insulte et crie tout le temps contre son mari, ses enfants, et son frère, souffle toute sa rancune contre tous. Elle insulte cette situation qui a fait d'elle une machine qui roule sans atteindre le but. Elle maudit son mari, mort, tandis qu'elle souffre.

Voilà tout ce que nous a laissé ton père, ce propre-à-rien : la misère. Il a caché son visage sous la terre et tous les malheurs sont retombés sur moi. Mon lot a été le malheur. Toute ma vie! Il est tranquille, dans sa tombe. Il n'a jamais pensé à mettre un sou de côté. Quant au frère, c'était plus simple: il ne mit jamais les pieds chez elle (la grand-mère). (Dib, 1952-1966, p.28)

Cette misère prend la forme de la détresse d'une femme, d'une mère et d'une fille. Tous les ressorts sociaux psychologiques et culturels se désagrègent. Mais une autre forme de misère se dégage de l'œuvre sur la condition humaine de Mohamed Dib : celle de la patrie.

\section{III- 2-3- LA MISÈRE. DE LA PATRIE}

Dans La Grande Maison, une autre misère se dégage ; c'est celle de la patrie, c'est-à-dire la terre algérienne usurpée par le colonisateur français. Par conséquent, on est devant deux personnages : le colonisé et le colonisateur, devant deux 
réalités opposées l'une à l'autre. Un agresseur qui a toutes les possibilités, se donnant complétement le droit d'exploiter, de massacrer, d'anéantir. Un agressé qui fait son mieux pour défendre sa vie, ses terres et sa patrie. Le colonialisme français, dit-on, est venu en Algérie pour mettre fin à la barbarie qui régnait parmi ces peuples selon leur conception, et qu'ils étaient venus pour sauver ces peuples. Mais la réalité est autre chose. Il ne s'agissait en effet que la politique expansionniste (Chaulet-Achour, 1985, p. 143) que tous les livres d'Histoire du colon enseignaient.

De la valeur civilisationnelle du colonialisme! Mohamed Dib refuse et récuse complètement cette pensée, insistant que le colonisateur n'est venu que pour effacer et détruire l'identité du peuple algérien. Par exemple la langue maternelle, [...] l'Arabe sera remplacée par celle du colonisateur [le Français]. Or la langue est la marque par excellence de l'identité d'un peuple. (Charaudeau, 2001, pp. 341348)

Mais pourquoi Dib a-t-il refusé cette pensée ? Parce que c'est à partir de la langue que l'homme conquiert le droit à sa propre existence dans le monde. La France désire marginaliser la langue arabe en établissant son système d'enseignement. Mais l'Algérie n'avait pas attendu le colonialisme pour savoir ce qu'était l'enseignement. "Après les transitions douloureuses que connut la société bouleversée, privée de ses biens nationaux, de sa liberté ou épreuve le besoin d'une culture ou plus exactement d'un enseignement même si cet enseignement s'était 
présente en Patagon ou en Zoulou, on l'aurait accepté.» (Calvet, 1974, p. 91)

L'appartenance à n'importe quelle communauté n'est assurée que par le biais d'une langue commune héritée d'une génération à l'autre, une langue homogène représentative d'une identité collective.

Charaudeau l'explique clairement en ces termes :

Elle est le lieu par excellence de l'intégration sociale, de l'acculturation linguistique, où se forge la symbolique identitaire. Il est également clair que la langue nous rend comptables du passé, crée une solidarité avec celui-ci, fait que notre identité est pétrie d'histoire.

(Charaudeau, 2001, p. 361)

Le colon a deux autres points de vue concernant cette question; le premier est que les pays colonisés tiraient un grand profit en apprenant la langue de l'occupant. Le colonisateur considère que c'est à partir de sa langue qu'ils peuvent être introduits dans le monde moderne. Le deuxième, selon les termes de Clavet, est que les langues indigènes seraient incapables de remplir cette fonction, incapables de véhiculer des notions modernes, des concepts scientifiques, incapables d'être des langues d'enseignement, de culture ou de recherche. En d'autres termes, explique L.-J. Calvet :

Cette manière de nier la langue des autres peuples, cette négation, avec d'autres, constituant le fondement idéologique de la supériorité de l'Occident sur les peuples exotiques [...] " va 
déboucher sur "le racisme et le colonialisme glottophage. (Calvet, 1974, p. 123)

La pensée coloniale vise toujours à diminuer l'importance des langues locales et ce, pour mieux assurer la propagation de la langue française et par conséquent le régime colonial serait imposé :

[...] le discours colonial sur la langue n'est pas seulement raciste, ou méprisant. Ce ne sont là que des manifestations superficielles. Il est avant tout et plus profondément fonctionnel. Tout tendu vers un but, la justification de la glottophagie et de la politique qui l'englobe. Car l'ablation des cultures locales, des langues locales, à quoi tend ce discours colonial, présuppose une culture et une langue de remplacement: c'est le mythe de l'assimilation. (Calvet, 1974, p. 165)

Par ces preuves ci-dessus montrées, l'écrivain veut nous transmettre une signification très importante quand il parle de l'école d'Omar: "Omar continuait à aller à l'école francoarabe » (Dib, 1952-1966, p. 29). Cela démontre que l'école n'était pas purement arabe mais mélangée entre l'arabe et le français. Ce qui prouve la puissance du colon qui a complétement réussi à étendre son influence non seulement sur la vie générale des Algériens mais aussi sur l'instruction. Cependant, se pose une question ; pourquoi Mohamed Dib at-il pu s'exprimer en français et non par la langue natale ? Dib répond à cette question par la citation suivante tirée de La Grande Maison pour montrer les agissements du 
colonisateur qui impose le français comme langue officielle dans l'enseignement scolaire du pays :

Paradoxe; une question, cette question qui émane chaque fois de Français-peu désireux il faut croire de se rappeler que l'Algérie a constitué des départements français durant près d'un siècle et demi et qu'ils y ont assumé le devoir d'instruction. (Dib, 1998, p. 191)

Toujours dans la misère de la patrie ; dans un des chapitres du roman, on trouve M. Hassan qui est dans une classe demande aux élèves :

Qui d'entre vous ce que veut dire: Patrie»?

Les élèves cherchèrent entre les tables, sur les murs, à travers les fenêtres, au plafond, sur la figure du maître; il apparut avec évidence qu'elle n'était pas dans la classe.

[...]

- La France est notre mère Patrie, annonça Brahim.

[Omar, surpris, pensait] Comment ce pays si lointain est-il sa mère? Sa mère est à la maison, c'est Aïni.

D'une voix basse où perçait une violence qui intriguait : ce n'est pas vrai, fit-il [Monsieur Hassan, l'instituteur] si, on vous dit que la France est votre patrie ».

(Dib, 1952-1966, pp. 17-31)

Cette phrase, dite par M. Hassen, considéré comme instituteur, autrement dit, fonctionnaire chez l'État français, est hautement politique. Cela dit que le régime colonial a 
continuellement utilisé l'école comme outil d'acculturation. La phrase de M. Hassen est expressive, significative à plus d'un sens. Elle (la phrase) est «tombée » en classe et reçue par les élèves comme un poignard enfoncé dans les cœurs. Il est à indiquer que $\mathrm{M}$. Hassen a dit cette phrase dans la langue de ses élèves, à savoir l'arabe, ce qui est strictement interdit. En d'autres termes, vu le contexte historique, il a couru le grand risque non seulement de perdre son emploi, mais aussi sa liberté pour voir son pays libérer. (Mansouri, 2012, p. 60) Il est mémorable de mentionner que Mohamed Dib a exercé la profession d'instituteur à Zoudj Beghal qui se trouve aux frontières algéro-marocaines entre 1939 et 1942 . L'instituteur est déchiré entre ses responsabilités d'enseignant; c'est-àdire de se conformer rigoureusement aux directives du Ministère de l'Éducation et son devoir d'Algérien, non seulement de ne pas accepter le mensonge, mais se l'interdit de le répandre en l'inculquant aux jeunes élèves qui ont foi dans la parole de leur maître d'école.

«Ce n'est pas vrai... » signifie la fausseté, le mensonge scolaire, "...si on vous dit que la France est votre patrie » (Dib, 1952-1966, p. 21). Il y a là, une démarcation nette de l'instituteur et un retour manifeste aux origines. La conscience algérienne commence à se développer progressivement et révèle le grand mensonge de la France ; Omar est troublé par le désordre qui l'entoure, particulièrement à l'école, critique spontanément le système éducatif imposé par le régime colonial français. L'instruction qu'il reçoit à l'école a quelque chose de faux. L'enfant est choqué scandalisé par ce système qui porte sur le mensonge, tromperie et falsification : 
On apprenait des mensonges pour éviter la fameuse baguette d'olivier. C'était ça, les études, les rédactions (...). Les élèves entre eux.

Disait : celui qui sait le mieux, le mieux arranger son mensonge est le meilleur de la classe (...) M. Hassan leur faisait des lectures où il était question d'enfants qui se penchent studieusement sur leurs livres.

La lampe projette sa clarté sur la table. Papa, enfoncé dans un fauteuil, lit son journal et maman fait de la borderie. Alors, Omar était obligé de mentir. $\quad$ (Dib, 1952-1966, p. 18)

La misère se trouve dans le système éducatif colonial qui conduit l'enfant à perdre confiance en ses instituteurs d'école. Il les considère comme ceux qui étouffent la vérité. Ils sont les instruments de l'ennemi, ils l'aident à réaliser leur objectif : c'est sous le contexte de la misère et la souffrance de peuple algérien pendant la période coloniale, et comment le colonisateur veut déchirer l'identité et la culture de ce peuple, et beaucoup plus marginaliser la langue mère, les traditions et les valeurs arabo-musulmanes (BADIS Rahima, 2017, p. 68). La position de l'instituteur Hassan, qui ne peut être que l'auteur lui-même, est incontestable. C'est le résultat d'une conscience politique, et pour la première fois, le professeur parle en arabe alors que les règlements intérieurs de l'école française l'interdit strictement. Ainsi, cette position de M. Hassan est vu comme un moyen de refuser ou même de détruire l'altérité pour avoir dit que « je reste toujours moi-même sans jamais être l'autre » (Dib, 1952-1966, p. 25) En d'autres termes, l'instituteur se serait considéré en porte-à- 
faux vis-à-vis de sa conscience en laissant ses disciples continuent à croire que la France est leur patrie. Il montre du courage sans un précédent pour leur enseigner une vérité d'une grande importance, hors programme.

Cela signifie le nationalisme. Même si la politique peut entrer dans les aspects cités plus haut, nous l'analyserons dans un autre cadre.

\section{III-3- LA MISÈRE, UNE POLITIQUE PARTICULIÈRE}

III-3-1- LA MISÈRE, UNE ARME POLITIQUE FRANÇAISE

Une question se pose encore :

Est-ce que la misère est une politique française pour l'humiliation et la torture contre le peuple algérien ?

La misère n'épargne personne. Elle est partout, dans Dar Sbitar, dans les rues, dans les écoles où les responsables ne s'intéressaient guère au confort des petits algériens qui devaient être contents de fréquenter l'école.

Mais c'était l'échec du pouvoir indigène à dialoguer avec le colon

De ces enfants anonymes et frileux comme Omar, on en croisait partout dans les rues, gambadant nu-pieds. Leurs lèvres étaient noires. Ils avaient des membres d'araignée, des yeux allumés par la fièvre. Beaucoup mendiaient farouchement devant les portes et sur les places. Les maisons de Tlemcen en étaient pleines à craquer, pleines aussi de leurs rumeurs.

(Dib, 1952-1966, p. 88)

(La grande misère des humbles gens ...)Dr. Amin Salah El-Din 


\section{III-3-2- LA MISÈRE, SANITAIRE}

S'ajoute à tout cela, la misère du manque de la santé à cause des maladies répandues dans toute l'Algérie que l'écrivain montre dans son roman : ces maladies sont étroitement liées à la sous-alimentation ;

La fièvre : "Les enfants frileux, les yeux allumés par la fièvre ». «Les yeux constamment dilatés avaient chez tout le monde un état fiévreux ». (Dib, 1952-1966, p. 149)

Le rachitisme : «De ces enfants anonymes, on en croisait partout. Leurs lèvres étaient noires. Ils avaient des membres d'araignée... » (Dib, 1952-1966, p. 28)

La tuberculose : «Ahmed Dziri, le père d'Omar, mourut d'un mal à la poitrine »...Djillali, le frère d'Omar, fut emporté par la même maladie ». (Dib, 1952-1966, p. 152)

L'auteur a pris conscience du rôle que la littérature pouvait jouer dans le mouvement national. Ce pourrait être la bonne base de construction contre la répression coloniale, pour exposer et dénoncer le drame algérien : «Les travailleurs unis sauront arracher cette victoire aux colons et au gouvernement général. Ils sont prêts pour la lutte ». (Nidiaye, 2004, p. 48)

La misère semble intégrale :

Il y a dans le bled des familles dénuées de tout que décime la misère physiologique et dont l'ultime unique idéal est de de pouvoir manger à leur faim. (Dib, 1952-1966, p. 123)

Cette Algérie française de l'époque d'écriture de cette ouvre est marquée par toutes les misères décrites par Mohamed Dib dans La Grande Maison. 


\section{CONCLUSION}

En conclusion, l'article sur le roman : La Grande Maison de Mohamed Dib, a permis de dégager des problématiques essentielles ; de l'espace et du temps d'écriture, la condition humaine et les manifestations de la grande «misère » dans cette œuvre d'anthologie. Cet espace, l'Algérie et le temps, la colonisation décrivent la situation sociopolitique, culturelle, éducative et sanitaire difficiles. Les sujets portent sur le destin de la population musulmane, pauvre et analphabète mais aussi sur la condamnation du colonialisme par les pères fondateurs : Mouloud Feraoun, Mouloud Mammeri et Mohammed Dib.

La combinaison espace et temps ont permis de dégager la condition humaine, mais aussi de voir les difficultés postindépendances. Cette condition humaine, c'est la misère, la faim, l'analphabétisme, les droits confisqués du peuple algérien. Cette étude est une véritable aubaine, une ouverture sur l'autre monde, une tentative pour museler l'obscurantisme. Sa richesse ! C'est un véritable témoignage sur la misère physique et morale, endurée par le peuple Algérien comme d'antres à cette sensible époque. Dib y parle de tout ce qui dégrade l'humain sans exception. Cette œuvre est un attachement et un devoir à sa communauté, avec un contrat social et, particulièrement, avec contrat moral.

Par conséquent, on peut dire que l'ouvrage de Mohamed Dib est une contribution à la formation du nationalisme algérien, à faire une sorte de mobilité sociale et politique dans la société algérienne dont il est originaire. L'écrivain, dans son roman, signale qu'une nation trop misérable et privée de la moindre subsistance est certainement prête pour la 
révolution. Ses écrits contribuent à former un nouvel esprit et pousse les Algériens à sacrifier par l'âme et par le sang pour leur «bled l'Algérie » qu'ils adorent beaucoup plus que leurs enfants et leurs familles.

L'œuvre est un apport universel et humaniste même si, elle est typiquement nationale. En somme, il est évident que Mohamed Dib n'écrit pas pour se faire ou inscrire son nom dans l'histoire littéraire. Son objectif essentiel : transférer et interpréter une douloureuse période historique dans la vie du peuple Algérien. C'est là une fin heureuse, un espoir donné pour un monde nouveau; et La Grande Maison est cet espoir pour tous. 


\section{BIBLIOGRAPHIE}

Abousedera, N. (1992), D.E.A point de vue et récit d'enfance dans la Grande Maison de Mohammed Dib, Paris 13, Charles BONN.

Arnauld, Jacqueline (1986): La littérature maghrébine de Langue Française, (2tomes), Paris, Publisud.

Amhisis-Oukel, D. (2012), Dar Sbitar, Une lecture de la Grande Maison de Mohamed Dib, Casbah éditions.

BADIS, Rahima (2017), Reflet des traditions et valeurs sociales dans La

Grande Maison de Mohammed Dib, mémoire de master, Université Larbi Ben M'Hidi.

Belhadj-Kacem, N. (1983), Le thème de la dépossession dans la trilogie de Mohamed Dib. Entreprise nationale du livre.

Bonn, C. (1986), Lecture présente de Mohamed Dib, Alger, E.N.A.L

Brahimi, D. (2001), Langue et Littérature francophone, Ellipses Éditions.

Calvet, L.-J. (1974), Linguistique et colonisation, Payot, Paris.

Charaudeau, P. (2001, mars). Langue, Discours et identité culturelle, ElaÉtudes de linguistiques appliquées, $N^{\circ} 123-124$.

Chaulet-Achour, C. (1985), Abécédaires en devenir: idéologie coloniale et langue française en Algérie, Entreprise algérienne de presse.

Déjeux, J. (1977), Mohamed Dib, écrivain algérien, Naaman.

Déjeux, J. (1979), La littérature Algérienne contemporaine, Paris, Presses universitaires de France.

Dib, M. (1952-1966), La Grande Maison, Paris: Editions du Seuil.

Dib, M. in interview (1958).

Dib, M. (1985, février 7), Témoignage Chrétien.

Dib, M. (1998). L'arbre à dires, Paris : Albin Michel S. A.

Dib, M. (Le 4 juin 1964.), Le Figaro littéraire.

Dictionnaire fondamental du français littéraire (2005), Forrest Philipe et Conio Gérard, la Seine.

Dictionnaires des Littératures : Larousse (1990), Paris, Larousse.

Guatarni, M. (2010), Mohammed Dib : la trilogie-Algérie: Un contrat social, Le Quotidien d'Oran.

Khadda, N. (1983), L'œuvre de Mohamed Dib, Alger, O.P.U.

Khadda, N. ( 1986), Mohamed Dib : Esquisse d'un itinéraire, Alger, O.P.U.

Khadda, N. (2003) , Mohamed Dib, cette intempestive voix recluse, Paris, Edisud, Aix-en-Provence. 
Lacheraf, M. ( 1988), Écrits didactiques sur la culture, l'histoire et la société en Algérie. Alger: Édition ENAP.

Mansouri, Y. (2012), L'ENGAGEMENT DANS “L'INCENDIE" de Mohamed Dib. Algérie: Université El hadj Lakhdar-Batna.

Nidiaye, C. (2004), Introduction aux littératures francophones, Presses de l'Université de Montréal, 2004

Remache, A. (2018, décembre), Panorama du Roman Algérien d’Expression Française ; Espaces et Espérances,. Synergies Algérie $n^{\circ} 26$.

Sartre, J.-P. (1948), Qu'est-ce-que la littérature?, Gallimard.

Volney, C. ( 1971), Les Ruines, Armand Colin.

Zima, P. (1985), Manuel de sociocritique, Paris : Picard.

\section{Sitographie}

1- http://www.fabula.org/document 4451.php

2- http://www.larousse.fr/encyclopédie

3- http://www.lettres.org/lexique/

4- http://www.lexpress.fr

5- http://www.limag.com 


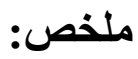

قبل طرح إثكالية جنسية الأدب الجزائري المكتوب باللغة الفرنسية ، و قبل الخوض في الموضوع ، يجدر بنا الإشارة إلى الأسباب التي أدت إلى الإزدو اجية اللغوية في الجزائر، و تمثلت في عدة عوامل من بينها التاريخية و الثقافية و الإجتماعية خلفتها بالدرجة الأولى المرحلة الإستعمارية التي حاولت طمس الثخصية الوطنية عن طريق محاربة اللغة العربية وتشريد المواطن الجزائرى من خلال اذلاله وتجويعه. تدور قصة هذه الرواية حول البؤس والتعليم الزائف الذي تديره المدرسة الفرنسية للأطفال الجزائريين الصغار. ومع ذلك ، ييقى الموضوع الرئيسي الجوع و البؤس. كان الطفل الصغير دائمًا جائعًا ، جائعًا بشكل رهيب ، لأنه لم يكن هناك ما يأكله في المنزل تقريبًا ، حتى أصبح عادة بالنسبة له (لم يتم إثباعه

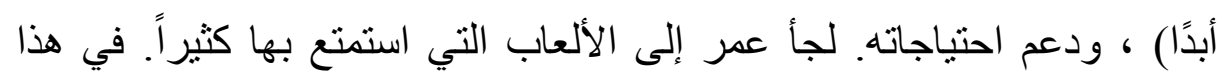
البؤس وبين جميع الثخصيات الذين يفكرون فقط في كيفية الحصول على ما يكفي من الطعام ، يضاف الي ذلك مأساة الوطن وفقد الهوية القومية الجزائرية. 